\title{
La dosimétrie sera-t-elle un frein au passage à la 3D ?
}

\author{
Jacobs R \\ Oral Imaging Center, Faculté de Médecine, Leuven, Belgique \\ reinhilde.jacobs@uz.kuleuven.ac.be \\ reinhilde.jacobs@med.kuleuven.be
}

L'imagerie constitue un des plus outils diagnostiques dans la pratique dentaire. Jusqu'à présent, les examens en 2D sont principalement employés pour visualiser les structures maxillofaciales. Ceux-ci ont néanmoins certaines limitations comparés à l'imagerie 3D. L'avènement de la technique de tomographie à faisceau conique (dit «cône beam CT») a remis en question le statut d'examens en 2D ainsi que le statut du CT-scan spiralé du massif maxillofacial.

Actuellement, le domaine du cône beam CT augmente très rapidement et cet examen trouve de nombreuses indications dans toutes les disciplines dentaires. Les arguments en faveur du cône beam CT seraient essentiellement une plus faible irradiation (Loubele et al. 2005, 2008, 2009) et une qualité d'image supérieure (Liang et al. 2009). Le cône beam CT est une technique récente qui remplacera probablement, le CT-scan maxillo-facial dans la plupart de ses indications. Néanmoins le CT-scan spiralé optimisé, en utilisant le protocole dit «low-dose » reste encore d'actualité pour les mêmes applications.

L'objectif de cette conférence est d'acquérir une vue sur l'utilisation du cône beam CT en pratique dentaire. Pour prendre des décisions précises sur l'imagerie 3D au moyen de cône beam CT, certains aspects doivent être abordés:

- quand un tel cliché est il indiqué ?

- comment prendre un cliché cône beam CT?

- comment interpréter les clichés cône beam CT?

- comment agir d'une façon responsable et justifiée avec l'irradiation d'un cône beam CT ? quels paramètres doit-on utiliser pour suivre les règles de radioprotection?

La dose efficace, exprimée en $\mu \mathrm{Sv}$, dépend de plusieurs facteurs, entre autres les constantes de tension $(\mathrm{kV})$ et d'intensité ( $\mathrm{mA})$, le temps de pose, le champ de vue, la qualité d'image envisagée. Pour la majorité des appareils cône beam CT, la dose reste inférieure à celle du CT-scan. Il y a quelques appareils, dont la dose efficace est seulement 2-4 fois plus élevée que celle des appareils panoramiques.

Un organisme européen (l'Association Européenne de Radiologie Dento-Maxillo-Faciale, www.EADMFR.org) a proposé des principes de base pour une utilisation rationnelle de la technologie «cone beam » dans les applications dento-maxillo-faciales (European Commission. Radiation Protection 136 2004, Horner et al. 2009).

En même temps, le projet SedentexCT de la commission Européenne (voir www.sedentexCT.eu) essayera d'optimiser la balance entre la qualité d'images et la dosimétrie des appareils Cone Beam $\mathrm{CT}$ ). Le but final est de réaliser une imagerie maxillo-faciale en $3 \mathrm{D}$, libérée du frein de la dosimétrie. 